\title{
Il passaggio dalla paura al riso nella trilogia di Palazzeschi Riflessi, Il codice di Perelà e La piramide
}

\author{
Prof.ssa Lamiaa AlyMosselhy Mohamed ${ }^{1}$
}

\begin{abstract}
While reading about science fiction, I found out that this terminology appeared in the early nineteenth century, but the characteristics of this genre were clear in Palazzeschi's work years before. Therefore I chose three of his romances Il codice di Perelà, Riflessi and La piramide. These three reflect the natural way that Palazzeschi used to express science fiction ideas. The writer discussed important matters regarding the identity case, the society challenges in the late eighteenth century and his attitude regarding the truth approved once and for ever. He also distinguished his stories by using a humorous style. His vocabulary and ideas were colored by his Tuscany origins. In addition to the introduction, the research discussed in its first parts how the writer's personality and mentality were formed. It also tackled the great philosophers and writers who influenced his ideas and style. As for the second part, the research underlined the most important cases which were concerned with the writer. Finally, I elaborated the effects of his local roots on his literary style.
\end{abstract}

Keywords: Palazzeschi, fantascienza, toscano, riso, Perelà, Riflessi, Piramide

\section{Introduzione}

Sostengo che la scintilla alimentata da Galileo Galilei evoluta si tra la fine dell'Ottocento e l'inizio del Novecento ha dato il via ad una svolta scientifica che ha, di conseguenza, influenzato la letteratura. Questo periodo storico si ritiene anche molto delicato perché testimonia sull'Italia ancora in boccio. I letterati e gli intellettuali italiani avevano premura ad elaborare opere che manifestano l'identità del loro stato unito e nel contempo contribuivano al progresso umano attraverso le loro idee creative.

Questo ritmo frenetico delle invenzioni, i nuovi orizzonti di studio e le scoperte astronomiche hanno avuto un forte impatto sul lessico dei versi. Nacque il nuovo genere letterario di fantascienza che verrà successivamente sviluppato dal cinema grazie all'utilizzo di mezzi tecnologici d'avanguardia. Quindi possiamo affermare che la scienza fu il nucleo di queste opere e del pensiero razionale che regolava tutti gli aspetti della vita dell'epoca oltre ad essere riconosciuto come l'unico criterio di giudizio. Il punto di partenza di questa tipologia era un'ipotesi, una teoria scientifica o un'idea che andava al di sopra della fantasia umana. Quest'ultima svolgeva il ruolo della guida che decodificava gli enigmi dell'animo umano e delle verità percepite prima in modo assoluto.

Con la diffusione della lingua inglese, francese e l'egemonia americana di questo genere, cercavo uno scrittore italiano del tardo ottocento; un letterato che è passato per il dibattito letterario tra strapaese e stracittà; cercavo qualcuno che era contemporaneo alle visioni decadenti, futuristi e antifascisti; ha provato anche l'amarezza dei momenti 
malinconici della prima e la seconda guerra mondiale ed ha scritto qualche opera che poteva essere classificata di fantascienza.

La ricerca mi ha portato alla scoperta di Aldo Palazzeschi, lo scrittore noto per le opere che lo rendono di maggiore spicco e tra $\mathrm{i}$ pionieri italiani di questo genere fantascientifico. Le sue idee si manifestano ormai 20 anni prima della nascita di questo termine apparso circa 1926 e tradotto in italiano verso gli anni cinquanta, però i suoi romanzi rivestono una grande importanza non solo perché sono precoci, neanche perché trattano tematiche tipiche secondo i criteri con cui si valutano o si giudicano opere di questo genere, infatti sono temi e quadri espressi con naturalezza.

I romanzi o i film di fantascienza trasmettono spesso i timori e le preoccupazioni degli esseri umani perché riguardano il futuro, l'ignoto, gli avvenimenti imprevedibili, nuovi luoghi o figure spaventose. Quando leggiamo Palazzeschi, invece, sentimenti come l'allegria e la contentezza riempiono i nostri cuori a differenza del dolore e del malessere che suscitavano opere dello stesso genere.

Fra la sua produzione letteraria ricca, tratteremo tre delle sue opere: Riflessi, La piramide e Il codice di Perelà perché in essi spiccano gli aspetti distinti della sua fantascienza e risultano chiari anche le cause che ha trattato e i suoi mezzi tecnici innovativi. Il primo capitolo è dedicato alla sua formazione dovuta non solo allo studio, ma arricchita dal periodo storico e il sistema sociale e regimi politici d'allora. Esaminiamo, del resto, la sua vocazione letteraria affinata dal viaggio e dalla compagnia di intellettuali e scrittori di vari orientamenti: decadenti, crepuscolari, futuristi, anti-tradizionali e avanguardisti. Non c'è dubbio che l'epoca che ha vissuto Palazzeschi testimonia, per altro, le riflessioni di grandi filosofi e nuove teorie scientifiche che hanno influenzato la sua mentalità e il pensiero dell'uomo e soprattutto i letterati e le persone colte e ben istruite. Palazzeschi trasmette anche queste prospettive fra le righe e discute la crisi conseguente di cui soffriva l'umanità, però cercava anche di affermarsi attraverso i punti di eccellenza che l'ho hanno reso tale e la sua visione autonoma e nuova rispetto ai suoi letterati del suo tempo.

Il secondo capitolo volge l'attenzione alle questioni che preoccupavano lo scrittore e come le ha illustrate attraverso idee e personaggi inverosimili. Lui non si abbandona al mondo della fantasia, bensì cerca di rompere il ritmo usuale del nostro mondo inserendo modelli surreali o incongruenti per inquadrare le tentazioni e difetti umani. L'autore non si lascia trascinare in un'atmosfera seria e triste, anzi vince il suo riso vince la sua paura. Lo scopo di questa sezione non è solo esaminare questi assi della letteratura palazzeschiana, bensì li proponiamo come i perni su cui si aggirano tante delle sue opere, specialmente la trilogia trattata dalla tesi. Quindi questa parte focalizza il modo unico e nuovo con cui lo scrittore ha elaborato questi maggiori argomenti delle sue tematiche: le contraddizioni di certe classi o individui; il tentativo perenne dell'uomo di rispondere alle interrogazioni assurde o oscure riguardante il carattere, i comportamenti e l'esistenza e l'atteggiamento adottato dallo scrittore nei confronti delle piaghe indagate dell'animo umano e della società.

Nonostante abbia vissuto durante il periodo storico del fascismo, nella nostra ricerca non abbiamo rintracciato idee pro o contro il regime, abbiamo dato più importanza alle idee connesse alla fantascienza e che toccano cause umane universali perché è, secondo me, il privilegio di Palazzeschi tra gli scrittori italiani contemporanei e quelli in poi sono diventati più conosciuti per il genere fantascientifico. 
Si conclude con lo studio di alcune tecniche narrative dello scrittore. Aldo Palazzeschi condivide con altri scrittori qualche tecnica come ad esempio l'autobiografismo e il simbolismo. Si può riconoscere qualche caratteristica di un dato movimento letterario del tardo Ottocento e tra quelli novecentisti, ma ci sono anche peculiarità comuni inevitabili sia per la sua formazione sia per l'atmosfera letteraria che implica il momento storico.

Individuare queste caratteristiche e associarle allo stile palazzeschiano non ci farebbe conoscere i tratti dello scrittore, perciò la tesi non perde d'occhio il volto identico di Palazzeschi che si vede nella struttura della sua opera fantascientifica, l'analisi sintattica della sua lingua, il lessico di alto registro non comune e l'autoironia che si prova quasi in tutte le sue opere. La tesi vuole legare le tecniche dell'autore al titolo per inquadrare come le ha impiegate per andare oltre la paura, il dolore e la critica usuale.

Il punto di partenza di quest'analisi punta sul personaggio fittizio e inverosimile che mette gli altri personaggi, simbolo degli esseri umani e cittadini italiani, vis a vis alle loro perplessità e contraddizioni. Il riso umoristico non prende di mira qualcuno né è un riso amaro che rende il destinatario provare simpatia verso un dato personaggio, ma è un atteggiamento che rifiuta di rassegnarsi alla realtà o le condizioni difficili, ma se ne burla. In definitiva l'obiettivo della tesi è affermare le radici di questo genere nella letteratura italiana attraverso la ricostruzione dei pezzetti caratteristici della fantascienza da Palazzeschi e come questo genere sia diventato uno strumento comunicativo efficace e divertente tanti anni prima dei mezzi tecnologici che meravigliano lo spettatore o lo rendono immerso in un mondo troppo materiale, stressante che accresce l'ansia e la paura.

\section{Palazzeschi tra formazione e autonomia}

Come è cresciuto Palazzeschi? 1885 era il punto di partenza per questo letterato che erudì volentieri su quasi tutte le correnti letterarie del tardo Ottocento e inizio del Novecento in Italia e in Europa senza abbracciare in modo assoluto un'unica tecnica, adottare una data visione. Si può riconoscere l'impatto chiaro del futurismo e il simbolismo così come si può verificare i pensieri e le tecniche di alcuni intellettuali d'allora le cui opere uscivano contemporaneamente alla pubblicazione della sua trilogia. In Italia citiamo Marinetti e Pirandello e in Europa si ricorda Nietzsche.

L'inizio risale ai primi anni del Novecento; il suo rapporto forte con Marinetti e la partecipazione ai manifesti futuristi. Lui, pur nutrendo i principi del movimento e pur trasmettendoli attraverso $i$ suoi scritti, mantiene spesso una sua posizione futurista. Mentre i futuristi esaltavano:

Il vibrante fervore notturno degli arsenali e dei cantieri incendiati da violente lune elettriche e il volo scivolante degli aereoplani, la cui elica garrisce al vento come una bandiera, Palazzeschi voleva INVECE DI FERMARSI NEL BUIO DEL DOLORE, ATTRAVERSARLO CON SLANCIO, PER ENTRARE NELLA LUCE DELLA RISATA. (Marinetti, F.T. e al., 1911, p. 197)

Le tre opere hanno in comune l'idea di un viaggio: un viaggio nel tempo verso il passato o il futuro, viaggio di un personaggio inverosimile o un viaggio verso l'ignoto. Il progresso scientifico e tecnologico si vede nelle sue opere attraverso la raffigurazione 
dell'uomo di fumo nel Codice di Perelà, la descrizione grottesca dell'amico di fantasia ne La piramide o nella città immaginaria in cui accadono le azioni in Riflessi.

Il fuoco purificatore è un'idea tipica futurista; è, come l'ha impiegato l'autore, la vena che ha nutrito Perelà nato e cresciuto nella cappa di un camino ed erudito da tre vecchie. È una creatura strana che andava improvvisamente a spasso in un paese anonimo. Perelà - come l'hanno chiamato i cittadini - rivela l'ipocrisia della società di quel paese immaginario prendendoli in giro. Perelà questo uomo di fumo mette in luce le catene delle tradizioni e dell'unico modo di pensare che implicano la vita sociale, le leggi dello stato o gli impegni del potere nella corte reale. Ne La Piramide invece l'io narrante soffre di solitudine e trova la consolazione nell'invenzione spiriti gemelli giganti e grotteschi che fanno la guida al protagonista. La comunicazione ridicola tra due creature di natura diversa rompe il ritmo monotono del narratore e dissipa i suoi timori in modo da indurlo a fare un viaggio nel futuro rievocando i dettagli delle città e i paesi prima di visitarle. In Riflessi lo scrittore fa il contrario e viaggia verso il passato tornando ad una città anonima e immaginando le azioni passate descrivendo i paesaggi, le sale e le camere del palazzo dove morì sua madre. Quindi la prospettiva avanguardistica si impone sulle tre opere palazzeschiane, ma la sua fantasia dilegua le tenebri del dolore attraverso un sorriso.

Insieme al filone futurista nelle opere, Palazzeschi non può fare a meno del simbolo che si manifesta attraverso il mistero che avvolge gli avvenimenti e i personaggi e nel loro tentativo inutile di definire una verità unica riguardante la loro crisi o il loro dolore o concernente le grandi questioni di cui si preoccupano come ad esempio la morte.

Perelà questo uomo di fumo è spesso muto. Le sue sembianze e il suo corpo inconsistente e il suo arrivo improvviso e ingiustificato al paese hanno accresciuto le interrogazioni riguardante la sua identità e le interpretazioni riguardante il suo ruolo e le sue capacità. Queste domande, esclamazioni e speranze mettono in evidenza la molteplicità della verità così come evidenzia gli oneri di cui soffrono questi uomini a paragone di quel che lo chiamo l'alieno "leggero" simbolo di libertà.

In Riflessi l'opera è divisa in due parti; nella prima il principe Valentino decide di entrare in uno stato di ibernazione, si richiude nella sua camera del palazzo materno, era imprigionato dalla sua paura, sospettava tutto quello lo circondava, ammirava dalla finestra monte, la luna e gli alberi. Lui usciva con fatica ogni notte per mandare una lettera in cui esprime $i$ suoi sentimenti legati in parte al clima e ai paesaggi, alle impressioni sottintesi delle due serve nel palazzo e interrogandosi o rivolgendo domande all'amico. La seconda parte è una lunga investigazione sull'assenza del principe. In entrambi le sezioni dell'opera la ricerca di una verità unica sia da parte del principe sia da parte degli inquirenti o dei conoscenti affermano la stessa questione; la molteplicità e l'impossibilità di accertare o accettare un solo punto di vista. Valentino è insomma simbolo dell'uomo in cerca del proprio io.

Ne La piramide la prima parte dell'opera è basata sulla definizione dell'amico tesoro. Il protagonista si interroga sul vero valore di questo tesoro, le sue caratteristiche e la possibilità di trovarlo. Questo monologo interrogativo riflette giudizi molteplici sull'amicizia così come simboleggiano la solitudine che infligge il parlante simbolo dell'uomo d'allora: 
In definitiva l'accettazione della varietà (e della differenza) è la via che permette il rifiuto della violenza insita nel tentativo di imporre al reale un significato esclusivo e, al contempo, la via che, nell'accettazione della contraddizione quale elemento inderogabile dell'esistere, informa di sé (e rende dunque possibile) quel passaggio dalla nevrosi all'allegria che Palazzeschi ha in seguito raccontato. (Cangiano, 2010, p.51)

Inseguendo la questione della differenza non manca l'influenza culturale di Nietzsche, il quale invita al dubbio ed esalta la diffidenza affinché si accerti dei valori stabiliti nella società o nella propria coscienza. Questo passaggio di diffidenza che deve affrontare l'uomo lo rende una creatura libera e nel contempo leggero come Perelà:

Perelà è l'archetipo dello Zarathustra nietzscheano per la qualità intrinseca della leggerezza e l'anelito a volare sopra la terra immonda. Zarathustra il lieve è detto da Nietzsche il suo eroe nel capitolo "Dell'uomo superiore". La mistica del superuomo si esprime con levità, dolcezza e mansuetudine.(Codice di Perelà, Zimarri, 2011, p.3).

La trilogia di Palazzeschi era quasi contemporanea alla produzione di alcune opere di Pirandello come ad esempio: Uno Nessuno Centomila. Appoggio che Palazzeschi ammirava la stessa idea dell'identità e la ricerca umana perpetua dell'io, ma lui si ravvisa per il metodo che adotta per identificarsi, cioè i suoi protagonisti tentano ad affermarsi attraverso l'altro.

Le dame della corte rivelano il loro vero carattere sfogando gli affanni con Perelà; parlano delle loro avventure di amore, dei loro desideri repressi o delle loro abitudini segreti e a volte vergognose. La verità soggettiva dovuta ai valori tradizionali e il livello culturale si nega attraverso Valentino che cerca se stesso interpretando gli sguardi e gli atteggiamenti delle serve e seguendo i cambiamenti accadutigli durante il suo viaggio di tempo al passato. Non parlava solo ad esseri umani ma discorreva con lo specchio, le porte, l'arredamento e gli ornamenti delle camere. Personificava i pagliai creandovi fantasmi indovinando, $i$ loro discorsi, nel loro conciliabolo parlano in malo modo [...], e ridono, uno è chinato all'orecchio dell'altro. (Palazzeschi, 1987, p. 39) Sembra che lui navigasse tra veglia e sonno e non si sa esattamente se le azioni fossero vere o meno. Questo perplesso accerta l'idea delle possibilità infinite. Questa ottica si afferma ancora ne La piramide. Il narrante coincidente con lo scrittore si trova attraverso gli amici inventati che scaturiscono proprio dal suo animo e dimostrano capacità straordinarie scambiando con lui qualche parola che rende la sua ricerca più chiara, ma non definitiva o unica:

Parvemi che... come un'ombra, nella rapida vertigine, un altro corpo uscisse dal mio corpo stesso. Inorridii. E subito mi vidi innanzi, e quasi addosso, un altr'uomo, un uomo come me, e forte, certamente, quattro volte almeno [...] -Via, su, avanti, su pollastrino bello, su gattino mio, fatti coraggio, su! [...] Come ti chiami? - Cecco [...] E mentre la mia mano si levava timida per cercare la sua e stringerla, mi assalse un brivido, simile a quello di quando Cecco era apparso, e sentii saltarmi addosso, come un-ombra, un altro corpo, e la mia mano ricadde vuota. (Palazzeschi, 1943, pp.559-562)

In questo brano spicca chiara l'idea del viaggio di tempo consistente nella vertigine che ha fatto uscire Cecco il gemello enorme del narrante che gli assicura il possesso di 
competenze superiori e lo induce a fidarsi di se stesso poi svanisce alla stessa maniera con un viaggio inverso di tempo.

Pur interessandosi della stessa causa dell'identità, Palazzeschi l'ha presentata in una forma innovativa di fantascienza facendo uso della navigazione nel passato e nel futuro. Lui l'ha, del resto avvolta con un sorriso. Il destinatario si diverte del giro invece di commuoversi o provare simpatia per il dramma del protagonista simbolo di tutti noi. È probabile che noi uomini siamo predestinati ad affrontare il dolore, la paura e la confusione, qualche volta ci si perde. Malgrado la sofferenza conseguente a questa sorte cui erano soggetti i vari personaggi di Palazzeschi, non si prova nelle sue opere un filone di pessimismo, anzi la conclusione delle opere è spesso aperta, coincide con la sua prospettiva della molteplicità di verità e interpretazione, il fine anche se non è definitivamente lieto costituisce uno sbocco e una speranza per un nuovo inizio.

\section{Tracce distinte della fantascienza palazeschiana}

Nonostante la fantascienza sia un genere letterario moderno, si considera una sottocategoria di un genere molto più ampio quello della "fantasia" (dall'inglese fantasy), cioè l'idea irreale è il nucleo dell'opera che viene associata ad una verità scientifica o ad un'ipotesi scientifica che potrebbe accadere al presente, al passato e spesso al futuro.

Il rapporto tra fantasia, scienza e tempo sono gli elementi che ravvisano opere fantascientifiche. Quindi bisogna prima individuare sotto quale categoria si possono classificare le opere oggetto di questo studio e quali sono gli obiettivi su cui punta l'autore nella sua opera fantascientifica. Innanzitutto quali sono gli aspetti di fantascienza nelle tre opere? Il punto comune tra le tre opere è il viaggio; potrebbe essere il viaggio di una creatura esterna al mondo terrestre, o di un uomo che vive in un paesino inesistente evocando la vita di un altro personaggio che ci viveva prima. Quest'avventura esplorativa prende di mira la società e la sua unità minima, l'uomo, è una fantascienza sociologica e nello stesso tempo umoristico.

Siccome la società è il perno della sua fantasia si riconoscono spesso la corte come classe nobile, la classe aristocratica e quella borghese. Nella corte il re, la regina, i ministri, i servi, le pressioni del potere, gli intrighi e l'animo che sta dietro a questa vita benestante. Nella classe aristocratica si impongono spesso le dame, i propri interessi e l'ipocrisia, invece nella classe borghese figurano personaggi come il buffo, il matto e il gobbo che cercano di superare le loro incapacità fisiche o mentali e coesistere con la società.

Gli avvenimenti accadono nella memoria del narrante, in un luogo generico di fantasia. Qualche volta l'autore esamina i protagonisti di tale società attraverso un personaggio che lo possiamo chiamare "l'alieno palazzeschiano", anche se l'invasione dello spazio è accaduta tanti anni dopo, una creatura inverosimile che non condividi la stessa formazione o luogo di nascita.

Io che mi sentiva così estraneo alla terra e attratto ancora alla sommità del camino, infilai inconsciamente le gambe in quelli stivali, e allora soltanto mi sentii sicuro, dritto, piantato, capace di poterci restare, lasciai la catena e incominciai a camminare (Palazzeschi, 1920, p.25)

Incontrava durante il cammino uomini che cercano di verificare la loro identità in maniere diverse, ognuno secondo la sua mentalità e livello culturale per poi venire 
circondato dalla classe borghese colta tra artisti e intellettuali e impiegati che accogliendolo, rivelano i loro interessi personali. Questo ceto sociale bada alla fama e alla gloria più alla conoscenza, ci trova l'opportunità che risponde alle loro esigenze e ambizioni; lo scultore voleva assicurare alla patria il suo monumento, per il pittore lui rappresenta il modello del suo capolavoro e il banchiere aspira a concludere con lui gli affari...ecc.

Il tempo più lungo lo passa con le dame simbolo della classe aristocratica. In questi incontri possiamo leggere il dialogo tra entrambi gli interlocutori anche se in realtà sembra un monologo che smaschera queste dame e smaschera il loro carattere vuoto:

Permettete signor Perelà che io vi lisci un pochino qui,

sopra un braccio. Sentite, sentite mie care, è morbido

più del più fino velluto, sentite. - Uh!

- Un velluto inverosimile

- Ma è sensazionale ! — È incredibile ! — Dio mio ! — Uhm ! — Che morbidezza ! — Ma sentite ma sentite': — Sentitelo qui, qui, qui.

— Siete tutto così ? - Un cigno. - Una nube mansueta.

- O uno di quei bei pennacchioni che saltano

fuori dalle locomotive.... - Ma sentitelo in questo punto ! - Ehi, sfacciata ! - Prima che bruciaste, signor Perelà, il vostro

vestito doveva essere di un magnifico velluto.

— Oh ! Rosso rubino ! Ardente come....

- Taci sciocchina. (Palazzeschi, 1920, p.47).

Queste dame, che dovrebbero appartenere alla classe più agiata che ha ricevuto un livello raffinato di formazione e cultura, sembrano donne pettegole, affascinate dall'aspetto di Perelà e i loro interessi appaiono superficiali.

Questa loro mentalità è dovuta al loro ruolo nella società, alle tradizioni e alle regole imposte nel loro paese, i quali riducono queste donne ad un livello inferiore, esse sono sottovalutate e non possono essere all'altezza degli uomini. Quindi diventano una risorsa di intrattenimenti che rispondono alle esigenze fisiche degli uomini:

signori uomini possono appostarsi sopra un piedistallo, col loro ingegno, colla loro scaltrezza, col loro denaro, a noi solamente la bellezza può dare un piccolo privilegio. La politica non ammette una sola pennellata del nostro colore sul suo quadro, la religione ci ammette solo per cornice

- Non possiamo celebrare.

— La scienza non ci appresta fiducia alcuna.... l'arte.... se non è quella del canto.... I signori uomini ci riserbano che facciamo scienza sì e no d'un po' d'amore, che loro ci richiedono poi come un passatempo.... o peggio ancora.(Palazzeschi, 1920, p.49)

Nonostante ciò questa stessa donna avvilita esercita le proprie arti in altri campi della vita e riesce ad addomesticare questa società maschilista attraverso i suoi giochi unici. La regina si ritiene il modello esemplare di questa categoria. In effetti il suo intervento nell'opera prevale su quello del re che si affaccia come un'ombra attraverso gli esponenti della propria corte e le firme sui decreti. La regina invece si impone come una donna esperta del gioco politico, ha avuto un lungo incontro con Perelà e al contrario delle dame che si interessavano di cose banali, la vediamo trasmettergli la propria esperienza e insegnanegli i principi del gioco delle dame con i re: 
Le dame della società ieri certo $v^{\prime}$ intrattennero allegramente, ma io....

io sono la Regina.... [...] io vi posso insegnare un giuoco però, un giuoco da Regina, il giuoco che si chiama dello Stato. [...]

- Prendete, ecco le carte, queste sono le dame, tenete, questi i cavalieri, li tengo io, qua le carte di spade. Mescolate le dame voi, io mescolo i cavalieri, mescolate le carte di denari, io le carte di spade. [...] quando il Re si combina colla carta più alta di spade muore. - E se non si combina ? - Finché non si combina regna. [...] Un nuovo Re, una nuova Regina, il denaro allo Stato, si rimescola il Re colle carte di spade finché non si combina colla carta più alta che rimane, la Regina raccoglie quella spada e qua, nel fondo della tavola. - Questo giuoco finisce ? - Questo giuoco non finisce mai. (Palazzeschi, 1920, pp.88.89).

Alla fine dell'opera cadono le maschere gentili di una grande parte di questi personaggi quando vengono chiamati come testimoni durante il processo di Perelà. Le stesse persone che lo apprezzavano e lo giudicavano geniale e di gusto raffinato l'hanno disprezzato e condannato a terribili fini. A questo punto si afferma la fantascienza sociale di Palazzeschi che ci tiene attraverso un suo alieno di mettere gli uomini di fronte ai loro difetti vergognosi, soprattutto all'ipocrisia dovuta ai dogmi politici o religiosi. Il contrasto tra l'ipocrisia dei cittadini e la leggerezza di Perelà rende chiara l'idea dell'uomo schiavo delle convinzioni e quello libero e autonomo:

- Formichini Cesare Augusto, scultore.

— Aveste rapporti coli' imputato ? - Si. — Che cosa vi sembrò ? - Un vile. — Ma voi gli avevate incominciato il monumento ? - Si. — E come mai ? - Già io feci agli eroi tutti il monumento.

- E ora incominciate a farne ai vili ? - Perchè più grande rifulga al confronto lo splendore

degli altri. - Credete si sia valso di male arti, ecc.... - Diaboliche. - Lo credete responsabile della morte di Alloro?

Satanicamente. - Credete ch'egli avrebbe continuata la sua propaganda, ecc. ?.... - Lucifero ! - Che cosa ne fareste ? - Prometeo ! (Palazzeschi, 1920, pp. 235,236)

Lo scrittore ha dedicato nell'opera Il codice di Perelà uno spazio più grande alle classi sociali e alla corte rispetto alle altre due opere. In Riflessi, dato le contemplazioni soggettive, il protagonista diventa l'unico rappresentante della classe reale. Il principe Valentino rompendo i limiti del tempo tratta la stessa causa della libertà e schiavitù. Lui è in continua ricerca della sua identità e mentre la ricerca, si trova costretto a un conflitto tra l'io schiavo e quello leggero tra il patrimonio culturale e formativo impostogli e la strada ignota che doveva percorrere per conoscersi:

"Ecco: io premo sopra una pesta accennata soltanto, e sento già di vivere la sua vita leggera, aerea, mentre preme ancora sopra di me come l'incubo da cui non riesco a liberarmi, il peso della mia" (Palazzeschi, 1987, p.43).

Questo principe si sente prigioniero, perciò ha deciso di ritirarsi nel palazzo reale di sua madre dove la paura se è impadronito di lui rendendolo una persona diffidente e incerta, ma questo stato l'ha spronato ad andare $<<$ senza direzione seguendo le gambe desiderose di movimento $>>$ e $<<$ per vie ignote senza pensare alle conseguenze $>>$. 
"Nel corso della sua strada ha cominciato ad interrogarsi: Come avevo potuto trovarmi su quella cima al di sopra della foresta?[...] dove ero? Ero sulla cima di quel monte così spesso contemplato dalla finestra della mia camera?"(Palazzeschi, 1987, p.131)

Ogni domanda lo scarcera dalla sua gabbia e al posto dell'incubo che incombe sul proprio animo, nasce un nuovo io che ha fiducia in se stesso che accetta la propria sorte e ne è essere cosciente :

"Ho pensato alla mia situazione del momento:di giorno avrei ritrovato la strada [...] ho pensato come in un esame della propria coscienza, coordinatamente a ogni vicenda della mia vita, saltutandone ogni passo.” (Palazzeschi, 1987, p.131)

Ne La piramide non ho individuato nessun trattamento per le classi sociali perché ci prevalgono riflessioni autobiografiche come simbolo dell'unità minima della società, l'uomo, ma nella seconda parte dell'opera l'autore dedica una grande parte ad un viaggio futuro a Venezia, la città reale descrivendo i suoi edifici, canali, gondole, negozi e paesaggi.

grandi esponenti.

Progetta anche di visitare Roma e richiama i fantasmi dei suoi della piramide, tiolo della sua opera e simbolo dei faraoni, sovrani di questa civita della piramide, titolo della sua opera e simbolo dei faraoni, sovrani di questa civiltà antica. Palazzeschi si dà l'avvio ad un viaggio futuro e inventa l'itinerario dell'escursione:

Partirò alla metà del mese, anzi, il giorno quindici precisamente.

Vi rimarrò una settimana. Sette giorni di una vita assolutamente eccezionale di una città straordinaria, di una originalità [...] Anderò; in gondola di giorno e di notte, lungo il Canal Grande [...] Il Quattordici del mese, quando tutto è all'ordine per la mia partenza, dopo aver vissuto un mese intero questo bel sogno di un soggiorno veneziano, su due piedi decido di non partire altrimenti [... ] Anderò invece, alla metà dell'altro mese a Roma e passerò a Roma una settimana di ricreazione invece che a Venezia.(Palazzeschi, 1943, pp.601-603)

Sembra che il protagonista abbia un'allucinazione, trasforma il sogno in una realtà stupefacente e avventurosa che sta al di sopra delle comuni descrizioni usuali di questi luoghi, è un rifiuto di ogni forma fissa che reprime la sua fantasia e la sua creatività. Strada facendo non si limita alla ricreazione del posto, gli pare:

nell'ombra di un'arcata, intravedere i fantasmi di un convito, e in mezzo ecco: Nerone, ebbro e panciuto, sozzo, a sganasciarsi dalle risate, il budello, che come tutti i figli dei grandi e dei saggi gode e disperde nel bagordo e nella follia la grandezza degli avi. (Palazzeschi, 1943, p.603)

L'autore condanna la vita agiata e superficiale dei nobili dell' antico impero romano raffigurando l'imperatore nella veste del buffo. Rapportando questa descrizione alle figure della stessa classe nelle altre opere, sembra che Palazzeschi accenna al loro deterioramento indicandone la perdita dei valori nobili che hanno radicato, una volta, degli antenati romani. Quindi Palazzeschi accentua nelle tre opere la crisi morale che c'era nel tempo descrivendo i comportamenti osceni e banali e subdoli di alcune dame o signori e nello stesso tempo il dilemma e la crisi di esistenza di questi ultimi, in continua ricerca a trovare una via d'uscita dalla vita cui sono costretti a coesistere.

In fin dei conti non si sa se lui avesse veramente visitato questi luoghi e incontrato questi personaggi. A lui non piacciono le realtà fisse e stimola lettori a non fidarsi dei pregiudizi 
o immagini relative ad un dato posto neanche alle sue riflessioni legate agli stessi posti, anzi, bisogna abbandonarsi alla propria realtà:

Oh! No ! No ! no ! e poi No ! Io fui a Venezia, a Roma è vero, ma il viaggio reale che vi feci è proprio quello che turba la mia immaginazione, le mozza il respiro, quello che io sono capace di fare [...] Ma se mai non foste in uno di codesti luoghi, dovete pure in qualche modo dare inizio alla vostra fantasia [...] Voi non potete figurarvi una Venezia nelle nuvole né una Napoli sotterranea, che se poi non sapete nulla potreste anche supporre che le piramidi sono delle carote [...] tutto serve a me come punto di partenza [...] Le piramidi sono fatte a punta [...] solo che la punta delle mie piramidi non è la vostra né precisamente quella dell'Egitto che non conosco [...] ma è una punta soltanto mia. (Palazzeschi, 1943, pp.620-622)

Da come lui si rapporta con queste città e paesi ci aiuta ad individuare un'altra caratteristica palazzeschiana di fantascienza ovvero la creazione di una realtà incoerente alla sua o a quella del destinatario. È una tendenza alla novità legato al tempo o al luogo come si vede ne La piramide in cui il protagonista sul bordo della sua macchina del tempo parte per città e paesi ben conosciuti, ma si diverte a parlarne come se fossero paesi delle meraviglie di cui nessuno mai visto o visitato. Il suo bagaglio culturale e le sue letture legate a questi luoghi alimentano la sua fantasia elaborandoli e presentandoli a modo suo. Si chiama il fattore straniante che nella letteratura fantascientifica, consiste in un'ambientazione empirica che non coincide con quella dell'autore e del lettore (o meglio, non coerente con il loro paradigma della realtà). (Iannuzzi, 2010-2011, pp.12,13)

\section{Le tecniche narrative}

All'inizio di questa ricerca abbiamo parlato degli intellettuali e le correnti letterarie e filosofiche che hanno contribuito alla formazione di Palazzeschi e hanno influenzato le sue idee. Passando alle sue tecniche narrative, ci corre l'obbligo di precisare la sua identità toscana che spunta in diversi aspetti tecnici narrativi.

Palazzeschi è uno scrittore fiorentino, le radici della sua città originaria si vedono nella presenza di uno o più personaggi burleschi. Questi personaggi, protagonisti o meno, sono spesso la personificazione di un oggetto o la descrizione di un comportamento umano astratto.

In Riflessi visto che il protagonista si isola in una sola camera della sua casa e vede il mondo da un'ottica ristretta, pensa che anche gli oggetti che lo circondano gli riservino rancore e inimicizia e lo deridono, perciò li presenta come buffi. Gli scambia il risentimento descrivendoli grottescamente. I pagliai, ad esempio, li vede goffi, storti, grotteschi, piccoli con la pancia ripugnante. Insomma sono Buffi! Buffi a vedermi dintorno. La buffonaggine non si limita solo alla deformazione fisica, ma include anche la scelta dei nomi come Copertino Enos che $<<$ pur portando un nome mascolino il teste rimane di sesso femminile. $>>$

Il personaggio inverosimile nelle opere di Palazzeschi è un elemento indispensabile, gli omosessuali, il gobbo e l'uomo di fumo ribadiscono l'idea della diversità e la lotta di queste creature a coesistere con la società e a superare il loro destino. 
Perelà e i propri comportamenti, commenti e gesti costituiscono il culmine della buffonaggine. A partire dalla scena della sua nascita, nella descrizione di un feto che cerca di auto liberarsi dall'utero nero del camino:

"Puntai le mani alle pareti e poggiando la schiena, e puntando le ginocchia, riuscii a scendere giù, dove il camino si allargava, lì incominciavano gli anelli di una catena, a quella mi aggrappai e discesi giù giù fino a terra." (Palazzeschi, 1920, p.25)

Quindi Palazzeschi sottolinea il legame con le arti figurative e con il genere «macchiaiolo» nella narrativa toscana della fine dell‘Ottocento, ripreso, come vediamo, dagli autori del Novecento: è ben evidente se si guarda ai «buffi» delle Stampe palazzeschiane, rappresentati in dimensione prevalente comica.(Yampolskaya,n.d., p.30)

L'impronta fiorentina spicca chiaramente nell'uso grammaticale di alcuni verbi soprattutto alla prima persona scrivendo mi sentiva, anderò, detterete o l'uso raro del dittongo uo come in cuoprite e scuoprite. Si nota anche l'uso dei derivati inusuali come facciona, vocino e Ceccaccio oltre all'uso di alcuni aggettivi come codesto, cotanto e tantino:

Nel lessico e nella fraseologia Palazzeschi sfrutta il fiorentino parlato, la lingua d'uso quotidiano. A questa base si aggiungono modi di dire gergali e termini tecnici, frasi fatte e, al contrario, parole rare e raffinate, voci disuete e popolari; è importante, però, che l'uso di tutti questi elementi è sempre ben motivato [...] il carattere più vero dell'elemento toscano si coglie nei modi di dire, nelle frasi proverbiali, numerosissime nella piramidell (ibid.) (arrotare le costole - darsi da fare; aspettare il Messia; ballar sopra un quattrino, ecc.). Non poche sono le voci d'uso fiorentino e toscano (fradiciume, piaccicone, zonzolare, ecc.). Anche nella morfologia si incontrano forme toscaneggianti (il congiuntivo sieno, l'imperfetto aveo, la seconda persona plurale voi andavi, ecc.). (Yampolskaya,n.d., pp.37,38)

In tanti casi il lessico e le forme sintattiche di Palazzeschi sono un pò difficili da capire soprattutto ne La piramide, il monologo e la descrizione dettagliata l'uso di colori per esprimere i suoi sentimenti positivi o negativi o che sono legati alle sue memorie o ai suoi sogni. Può darsi che questo uso prevalente delle ipotassi sia dovuto alle lunghe riflessioni unilaterali del protagonista o dei personaggi.

Nonostante ciò i dialoghi rispecchiano la lingua fiorentina economica ed ironica. Questi discorsi sono spesso finalizzati spesso a prendere in giro un personaggio di una certa classe sociale o per trattare umoristicamente una situazione come quando Perelà ha visitato il convento:

— Ecco suor Mariannina Fonte penitente. — Quante volte peccaste suora Fonte ? Un dì tre volte, signor Perelà. — Ed ora voi dimandate sempre perdono del vostro peccato ? - Ogni dì tre volte. — Ed ecco suor Colomba Mezzerino. — Penitente ?

- Peccatrice ella non è, signor Perelà, suor Colomba

portò qui il fiore della sua purezza e lo conserva,

ella prega per i peccatori. - Vi sono dunque due specie di persone, quelle

che dimandano perdono dei propri peccati e quelle

che implorano per i peccati altrui ? - Ed un'altra specie signor Perelà, quelle persone

che peccano solamente. Per quelle suor Colomba eletta

prega. Andate andate suor Colomba ad implorare per 
quelle persone. (Palazzeschi, 1920, pp.126,127)

Inoltre l'uso del dialogo riduce l'opera in alcune parti ad un testo teatrale, questa tecnica si vede nelle didascalie che accompagnano o precedono il dialogo o nell'intervento del coro, le voci della folla, i fischi, le parolacce o nelle esclamazioni:

Apre un giornale e piano piano lo distende, si mette a cantarellare, schiocca la lingua con una flemma da far morire. Io mi sento ardere, ho i vapori alle tempie, non ne posso più, sto per crepare .... oh! Dio

-Merda

- In bocca a te.Dalla tua bocca non poteva uscire altra cosa.

- Per venire nella tua

-bella educazione, raffinata, carino davvero, sì, speciale.

- Non ti credere mica di intimorirmi eh, insetto pornografico.

- Ma neppur tu a me, grosso come sei, pincone. (Palazzeschi, 1943, p.512)

Ritornando al titolo della ricerca potrei dire che le tecniche dello scrittore hanno in comune il riso come un elemento indispensabile, le questioni trattate nelle sue opere sono umoristiche quanto tragiche. Sono serie questioni umane che hanno disturbato tanti intellettuali, commediografi e autori, ma Palazzeschi si distingue per il suo metodo. Non solo cerca di sopravvincere il dolore, ma di trasformare il suo dramma in un viaggio divertente e piacevole e prova a rispondere ai quesiti e alle sue esigenze.

L'enigma dell'identità che confonde tanti dei suoi protagonisti e di cui si occupano tanti degli individui di una certa società o comunità si scioglie attraverso un discorso umoristico e qualche volta sarcastico, lasciando infine la risposta aperta per soddisfare tutti gli interlocutori. Non diventa più una questione di sofferenza, anzi è molto piacevole e interessante:

Pena! Rete! Lama! Pena! Rete! Lama! Pe....

Re... La.... - Ehi, galantuomo, dove andate ? - Alla città. — Ci sapete dire un po' che razza di bestia siete ? - Io sono.... molto.... un uomo. - Voi siete poco un uomo, di uomo mi sembra non

abbiate che le scarpe. - Di dove venite ? - Di lassù. - Bel discorso, ehi galantuomo, lo sapete con chi

parlate ?(Palazzeschi, 1920, p.12)

Questo alieno, sceso dopo la morte delle tre vecchie che l'hanno erudito leggendo sotto il camino, gli parlava alla stessa leggerezza del proprio corpo, parlava con naturalezza però il suo intervento rivela umoristicamente la banalità della società quanto la sua buffonaggine:

— Due fanciulle! - Veneziane! — Erano venute qui ad infilare le perle alle dame della città. - E per amore hanno troncate le loro giornate. - Amavano uno stesso uomo ? — Sì, signore. - E perchè si sono gettate nel pozzo ? — Bella, perché erano infelici. Come poteva egli con un cuore solo corrispondere a due cuori così ardenti ? E allora una sola doveva gettarsi nel pozzo. - Tacete, cosa sapete voi ? - Chi siete ? Una sola! Che faccia! - Mandatelo via, fatelo andar via! - Non vedete che uomo buffo ?(Palazzeschi, 1920, p.12) 
Lo stesso vale per il dramma della solitudine di cui soffriva il protagonista ne La piramide, il quale invece di rassegnarsi, la incarna in un amico straordinario e scambiano insieme giochi e battute:

Ma Visto che io rimanevo piantato lì, quasi di sale, senza la forza necessaria per andare né avanti né indietro, prese a misurare dall'alto la punta del suo dito sopra la mia testa e, quasi che il dito fosse calamitato e io di ferro, mi sentii sull'istante tutto girare[...] Per; mentre frullava e rifullava così la mia persona, come una trottola, una cosa mi era dato capire nella confusione grandissima [...] una cosa soltanto ti chiedo prima di rientrare al completo, dimmi almeno il tuo nome [...] Io non udivo ancora la sua voce che certo era in cammino chi sa mai da quanto[...] - Come ti chiami? - Agesilaaaaaaaoooo. - Pervenne alfine la remotissima voce salita da un pozzo senza fondo, e spargendosi nell'aria ripeteva concentrica: ao ao ao..

-Agesilao! Che nome carino! Agesilaoaoaoaoao ... non mi scorderò mai di te, mai ai miei giorni, tu mi hai levato un gran macigno dallo stomaco, ci avevo una colonna di granito, e ora mi sento vuoto, che leggerezza! Addio, addio sai, Agesilao, Agesilaoaoao ... Agesilaino .... Agesilauccio...(Palazzeschi, 1943, pp.575-577)

In Riflessi l'umorismo crea un'atmosfera comica basata sul senso contrario consistente nella moltitudine di ipotesi riguardante l'assenza del principe, sono giudizi infiniti che portano all'impossibilità di arrivare alla verità anzi di considerare la questione come una burla fatta apposta da Valentino:

Il principe Valentino Core non si sarebbe esploso il colpo di revolver alla tempia ma bensì al cuore. [...] il principe Valentino Core non sarebbe ancora morto ma si troverebbero, pertanto, in condizioni disperate [...] si conferma la smentita del suicidio del principe Valentino Core, egli sarebbe partito per ignota destinazione. [...] Il principe Valentino Core sarebbe vittima di un assassinio [...] è da escludere nel modo categorico l'ipotesi di un assassinio.[...] si accredita sempre di più l'ipotesi di una burla, una inesplicabile burla da parte del Principe. (Palazzeschi, 1987, pp.146-159)

Lo scrittore condanna umoristicamente, attraverso questo stato d'incertezza, la soggettività e le voci prive di fondamento che rovinano la società e la confondono di più, dal momento che è incapace di specificare una verità unica e certa.

\section{Conclusione}

In questa ricerca e nei capitoli precedenti ho studiato la formazione, le idee e le tecniche palazzeschiane per verificare come l'autore del tardo Ottocento avesse elaborato idee di fantascienza già prima della nascita di questo genere si sarebbe affermato nel mondo.

Ho estratto le caratteristiche di questo genere in tre opere: Riflessi, Il codice di Perelà e La piramide. In Riflessi e ne La piramide spicca chiara l'idea del viaggio del tempo che si vede ad esempio ne La piramide nella vertigine autonoma e violentissima cui era esposto il protagonista facendo nascere dal proprio corpo uomini straordinari. Questa idea somiglia alla macchina del tempo in cui navigano i protagonisti ed incontrano altri uomini di un'altra epoca. La stessa idea si vede in Riflessi in cui il protagonista fa un viaggio di tempo inverso al passato sulle orme della vita di sua madre per identificarsi. 
Ne Il codice di Perelà si presenta l'uomo di fumo che ho chiamato "l'alieno di Palazzeschi" in quanto incarna la stessa idea nelle opere e i film di fantascienza. Una creatura strana o extraterrestre di un aspetto umano che arriva sulla terra da un pianeta ignoto e durante il suo viaggio esplorativo si comporta qualche volta in modo buffo, parla come i bambini in modo spontaneo mettendo in imbarazzo gli altri o mira a salvare quella parte di mondo dove è atterrato. Altre volte si focalizza sui difetti individuali o convinzioni sociali sbagliate.

Perelà ha avuto il nome delle iniziali delle tre signore anziane che l'hanno istruito e ripeteva i loro nomi, è un uomo di fumo, un feto cresciuto dal fumo nell'utero nero del camino e che nasce in modo autonomo e scende sulla terra illustrando l'idea della leggerezza e il rifiuto di tutto ciò è tradizionale o obbligatorio e che rovina la vita umana. In Definitiva Palazzeschi sarebbe uno scrittore innovativo che non ha solo presentato idee fantascientifiche, ma propone anche una prospettiva sua per affrontare le questioni umane e sociali dell'epoca e ravviva tutto ciò con la sua tecnica umoristica toscana che lo rende unico tra gli scrittori del tempo.

\section{Bibliografia}

Atti del convegno internazionale (2002), L'opera di Aldo Palazzeschi, Firenze, Italia: Leo S. Olschki.

Boitani, P., Collini, P., Cronk, N., Del Lungo, A., DonnaRumma, R., Fedeli, P., ...Sestito,M.,( 2015), Modi di ridere, forme spiritose e umoristiche della narrazione, Pisa, Italia: Pancini Editore.

Cangiano, M.(2010), L'uno e il molteplice nel giovane Palazzeschi(1905-1915), Firenze, Italia: Società Editrice Fiorentina.

Febbraro, P.(2007), La tradizione di Palazzescbi, Roma, Italia: Alberto Gaffi editore.

Iannuzzi,G.(2010-2011), Letteratura fantascientifica Italiana, Un percorso tra istiturioni e testi, Dagli anni cinquanta agli anni settanta (tesi di dottorato),Università degli studi di trieste, Trieste.

Marinetti, F.T., Buzzi, P., Palazzeschi, A., Cavacchioli, E., Covoni, C., Altomare, L., ... Jannelli, G., (1911), I manifesti del futurismo, Firenze, Italia: Edizioni di "Lacerba".

Palazzeschi, A.(1987), Tutte le novelle, Milano, Italia: Mondadori.

Palazzeschi, A.(1943), Romanæi straordinari 1907-1914, Firenze, Italia: Vallecchi.

Palazzeschi, A.(1920), Il codice di Perelà, Firenze, Italia:Vallecchi Editore.

Yampolskaya, A.(n.d.), Lingua e stile di Aldo Palazzescbi:in confronto con le tradurioni russe (tesi di dottorato), Università degli studi di Firenze, Firenze.

\section{Sitografia}

Zimarri, E., (2011, April). Codice di Perelà. Retrived from http://www.la-ginestra.com/wpcontent/uploads/2011/04/zimarri-scar.pdf 